\title{
Heart rate variability interventions for concussion and rehabilitation
}

\author{
Robert L. Conder ${ }^{1}$ and Alanna A. Conder ${ }^{2}$ \\ 'Department of Sports Neuropsychology, Carolina Neuropsychological Service, Raleigh, NC, USA \\ 2 Pediatric and Sports Neuropsychology, Carolina Neuropsychological Service, Raleigh, NC, USA
}

Edited by:

J P. Ginsberg, Dorn VA Medical

Center, USA

Reviewed by:

J P. Ginsberg, Dorn VA Medical

Center, USA

Paul Comper, University of Toronto,

Canada

Leah M. Lagos, Leah Lagos LLC, USA

*Correspondence:

Robert L. Conder, Department of Sports Neuropsychology, Carolina Neuropsychological Service, 1540 Sunday Drive, Suite 200, Raleigh, NC 27609, USA

e-mail: bconder10@gmail.com
The study of heart rate variability (HRV) has emerged as an essential component of cardiovascular health, as well as a physiological mechanism by which one can increase the interactive communication between the cardiac and the neurocognitive systems (i.e., the body and the brain). It is well-established that lack of HRV implies cardiopathology, morbidity, reduced quality-of-life, and precipitous mortality. On the positive, optimal HRV has been associated with good cardiovascular health, autonomic nervous system (ANS) control, emotional regulation, and enhanced neurocognitive processing. In addition to health benefits, optimal HRV has been shown to improve neurocognitive performance by enhancing focus, visual acuity and readiness, and by promoting emotional regulation needed for peak performance. In concussed athletes and soldiers, concussions not only alter brain connectivity, but also alter cardiac functioning and impair cardiovascular performance upon exertion. Altered sympathetic and parasympathetic balance in the ANS has been postulated as a critical factor in refractory post concussive syndrome (PCS). This article will review both the pathological aspects of reduced HRV on athletic performance, as well as the cardiovascular and cerebrovascular components of concussion and PCS. Additionally, this article will review interventions with HRV biofeedback (HRV BFB) training as a promising and underutilized treatment for sports and military-related concussion. Finally, this article will review research and promising case studies pertaining to use of HRV BFB for enhancement of cognition and performance, with applicability to concussion rehabilitation.

Keywords: heart rate variability, concussion, mild TBI, biofeedback, neurofeedback, rehabilitation
Heart rate variability (HRV) has emerged as an essential component for study, research and clinical applications in physiology and psychophysiology (Moss et al., 2013). Research has shown implications for HRV in physiology, pathophysiology, psychology, psychopathology, cognition, and neurocognitive impairment (Gevirtz, 2013). Within the physical domain, HRV has been postulated as a measure of not only cardiac health, but of cardiac pathology and as a marker of possible mortality from cardiopathology (Bigger et al., 1995). Psychologically, given its connections with the autonomic nervous system (ANS) and limbic system, HRV can be a marker for anxiety disorders, such as a generalized anxiety disorder (GAD), post-traumatic stress disorder (PTSD), or a general predisposition to react sympathetically to external or internal stressors (Blechert et al., 2007). Cognitively, persons with high HRV have shown superior performance on neurocognitive measures of attention, concentration, working memory, and executive functioning (Hansen et al., 2003). Alternatively, HRV can be adversely affected by concussions or any degree of traumatic brain injury (TBI; Goldstein et al., 1998). However, there is research that HRV and HRV impairment can be modified and trained through exercise (Hedelin et al., 2001; Hansen et al., 2004; Hautala et al., 2009), diet (Lima-Silva et al., 2010), and biofeedback interventions (Lehrer et al., 2013). This article will first review theoretical models of the heart-brain ANS interaction, with specific emphasis on the adverse effects of sport and military concussions on HRV. Second, this article will review studies suggesting that heart rate variability biofeedback (HRV BFB) is a promising intervention for treatment of sport and military concussions.

\section{EPIDEMIOLOGY AND DIAGNOSIS OF TRAUMATIC BRAIN INJURY}

First, it is necessary to review the etiology and severity of TBI to understand where concussions fall along the continuum of severity of injury and how they may affect HRV. TBI is defined as an injury to the brain resulting from blunt trauma to the head or body, or acceleration or deceleration forces transmitted to the brain (Barr and McCrae, 2011). TBI severity is classified as Mild, Moderate, or Severe based on degree of injury severity, including presence and length of loss of consciousness (LOC) and number and degree of post-traumatic symptoms. These three categories are based on assessment with the Glasgow Coma Score (GCS; Teasdale and Jeanette, 1974). The GCS measures a patient's level of functioning based on eye opening and verbal and motor responses, resulting in a score ranging between 3 (minimum) and 15 (maximum). The Mild GCS group has a score of 13-15, and typically will have minimal or no permanent neurologic sequelae, with typical recovery expected in 1-3 months (Levin et al., 2012). The Moderate TBI group has a GCS score of 9-12 and may have permanent sequelae impacting personal life, school or 
work; finally, the Severe TBI group has a GCS score of 8 and below, and will almost always present with permanent neurologic damage at a moderate level or greater, affecting all aspects of life. It is the "Mild" group that is the primary focus of this article, as these are the athletes and soldiers that most often will be seen clinically and who may have cardiac correlates of concussion sufficient to interfere with cognitive and cardiovascular resources needed for athletic and military performance. Generally, patients with a GCS in the 13-15 range will not have LOC, but may have episodic cognitive confusion, transient amnesia, dizziness, slow reaction time, and balance problems (McCrory et al., 2013). When assessed in the Emergency Room, the traditional physical neurologic exam will be negative and non-focal for pathology, as will the Head CT. The traditional Emergency Room concussion evaluation protocol may not elucidate underlying neuropathology, which is being seen in controlled research studies of athletes with sophisticated neuroimaging including magnetic resonance spectroscopy, fMRI, or diffusion tensor imaging (DTI; Bluml and Brooks, 2006; Pardini et al., 2011) or neuroelectrical assessment including EEG and ERP (Broglio et al., 2009; McCrea et al., 2010; Barr etal., 2012). If someone with a presumed Mild TBI does present with greater neuropathology, such as a basilar skull fracture, intracerebral bleed, or cerebral hematoma, then they are generally referred to as a "complicated" Mild TBI and the severity of injury is noted, with implications for a more complicated recovery.

Giza and Hovda (2001) have elucidated an animal neurometabolic model of mild TBI or concussion, in which there is a significant mismatch between glucose metabolism and regional cerebral blood flow, with a concomitant influx of glutamate and other ionic changes in the cell membrane. Their studies have repeatedly shown a return to baseline neurometabolism around seven days post-injury, presumably without permanent cellular damage. These animal studies have provided the basis for predicted return to baseline functioning in athletes in 7-10 days. This neurometabolic model may be useful to explain the quick recovery typical of non-refractory concussions. However, refractory concussions (the focus of this article) may be better explained by a model of neuronal deformation induced by the biomechanical force of the injury (Abolfathi et al., 2009).

McCrory et al. (2013) postulate that concussions may overlap with the lower end of the Mild TBI spectrum, and may extend to a lesser degree of severity labeled Minimal TBI. The terms "concussion" and "Minimal TBI" may be used interchangeably and as an alternative to Mild TBI but "concussion" will be used in this article, consistent with its usage in sports medicine.

Bigler (2008) points out that a concussion from a football tackle, automobile accident or blast injury does not solely affect the brain. The concussive forces can affect all organs, including the heart (Cernak and Noble-Haeusslein, 2009). Cardiac sequelae of a primary blast injury may produce arrhythmias, ischemia or a myocardial infarction (Garner and Brett, 2007), even in the absence of cerebral concussion. For example, comotio cordis is an unfortunate and often fatal cardiac event from a direct blow to the chest wall, usually in baseball, and without a concomitant cerebral concussion. Additionally, Palma and Benarroch (2014) reviewed non-traumatic cerebral illnesses which impair
HRV, including epilepsy, ischemic, and hemorrhagic stroke and neurodegenerative diseases, such as Parkinson's. They postulate that these neuropathologies involve the insula, basal ganglia, and brainstem and may impair HRV, leading to secondary cardiac illness.

While there have been studies measuring actual G-force on the playing field (Guskiewicz and Mihalik, 2011), few such studies have been done for the most common cause of concussion in adults: automobile accidents. In children, the most common cause of concussion presenting at emergency departments is bicycle accidents (Gilchrist etal., 2011), followed by sport-related injuries. The change in momentum (impact) and transfer of kinetic energy in low impact auto accidents can produce a concussion, and these kinetics and V-max are presumed to produce greater forces than those experienced on the playing field.

Other factors that can impact concussion recovery include protective health status and motivation. Athletes and soldiers tend to have greater cardiovascular and aerobic fitness, which has been postulated as a protective factor for recovery from complicated concussion (Kontos et al., 2006), as opposed to the general, sometimes deconditioned, population. Psychosocial factors, such as motivation, also can impact recovery trajectory. Ponsford et al. (2000) tracked emergency room TBI admissions by mechanism of injury (MOI). After three months, the athletes who presented at the emergency room were significantly less symptomatic than those who presented with a motor vehicle accident as their MOI. Data is not currently available on quantification of blast injury for soldiers in theater, or their outcomes regarding concussion. Nevertheless, typical sequelae of a refractory Mild TBI include problems in attention, concentration, working memory, and executive functioning. Even though the MOI is different between blast injuries (Garner and Brett, 2007) and athletic injuries, the neuropsychological outcomes are similar (Belanger et al., 2009; Cooper et al., 2012) and symptom differences may be due to PTSD in the military group (Lippa et al., 2010). Tan et al. (2009) report a complex, synergistic relationship between PTSD, pain, Mild TBI, and HRV in OEF/OIF veterans.

\section{HEART RATE VARIABILITY}

As this article focuses on the relationship between HRV, cognition, and concussion, an explanation of HRV is necessary. While a full explication of HRV assessment and treatment is beyond the scope of this article, for our purposes we are interested in the measures traditionally cited in the research literature that are amenable to measurement and intervention in the clinic and in exploring the cardiac correlates of concussion. HRV is measured as part of the cardiac QRS complex. The Inter-Beat Interval (IBI) measured between R-waves is the basis for measurement of HRV. HRV was initially felt to be more artifacts, but now is itself the focus of intense study (Lehrer, 2013). HRV is also useful in that it can be measured with minimal hardware and software. A traditional EKG with as few as three chest leads or a three-lead wrist placement with electrodes can be used (Thought Technology, Montreal, Canada). A Blood Volume Pulse sensor (photoplethysmograph) attached to the distal phalange of a finger or the earlobe can be used with appropriate hardware and software to also calculate the IBI (HeartMath, Boulder Creek, CA, USA; StressEraser, New York, NY, USA). 
There are two standard metrics for measuring HRV for analysis and treatment. One is the time domain (changes over time) and the other is the frequency domain (measurement of a spectrum of oscillatory components of the heart). While there are multiple measures of time domain, the most common used statistical method is measuring the Standard Deviation of the Normal-toNormal interval (SDNN). The SDNN is basically normalization of the standard deviation of the R-to-R interval, with artifacts removed. Comparisons of data should be with equal epochs, usually $5 \mathrm{~min}$ for SDNN. The power spectrum measurements are fractionations of all the oscillatory frequencies contained within a specific epoch. The frequencies are generally calculated over the same epoch as the SDNN metric. There are three frequency subbands of particular interest within HRV. The very low frequency (VLF) is composed of frequencies less than $0.04 \mathrm{~Hz}$. The low frequency (LF) is composed of frequencies between 0.04 and $0.15 \mathrm{~Hz}$. The High Frequency (HF) band investigates frequencies between 0.15 and $0.4 \mathrm{~Hz}$. There are both physiologic and cognitive correlates of these frequency bands. The VLF band is considered to reflect mainly sympathetic activity. The LF band is considered to show parasympathetic activity, as well as the baroreflex and enhanced cognitive processing. The HF band is considered to reflect respiratory sinus arrhythmia, and activity from the vagus nerve (Combatalade, 2010).

These same hand-held HRV monitoring devices can provide visual and/or auditory feedback to train HRV. HRV training begins with breathing exercises to increase respiratory sinus arrhythmia, and help one find their resonant frequency - the frequency at which greatest HRV occurs. Breathing rates between 4.5 and 6.5 breaths per minute will produce the greatest HRV in most persons. The biofeedback instruments can provide a visual pacer for breathing rates and informs the person when they have achieved the greatest coherence between respiratory rate and heart rate. A more detailed training protocol is reported by Lehrer et al. (2000).

\section{NEURAL RELATIONSHIP BETWEEN BRAIN AND HEART}

Thayer etal. (2009, 2012) and Thayer and Lane (2009) have postulated an intricate Neurovisceral model of the relationship between the prefrontal cortex of the brain and the heart. In the Thayer model, prefrontal brain areas, including the orbitofrontal cortex and the medial prefrontal cortex tonically inhibit the amygdala, with disinhibition of the central nucleus of the amygdala. The deactivation of inhibitory nuclei leads to a net increase in sympathetic activity which eventuates in decreased HRV and increased heart rate. The central nucleus of the amygdala is believed to be the major efferent source of modulation of autonomic, endocrine, and cardiovascular responses. Three routes are postulated. One involves the activation of tonically active sympathoexcitatory neurons of the rostral ventrolateral medulla due to decreased inhibition from neurons in the caudal ventrolateral medulla, resulting in an increase in sympathetic activity. The second involves inhibitory neurons in the nucleus of the solitary tract, which can lead to a decrease in overall parasympathetic activity. The third postulated pathway involves direct excitation of sympathetic rostral ventrolateral medulla neurons, further leading to an increase in sympathetic activity. The overall result, regardless of the pathway, would be an increase in sympathetic output and overall heart rate, with a concomitant decrease in HRV. While these pathways have not been validated either electrophysiologically or with physiologic staining techniques, Lane et al. (2007) used positron emission tomography (PET) to measure medial prefrontal activity along with simultaneous measurement of spectral HRV. In these studies, subjects were shown film clips depicting emotional situations, involving happiness, sadness, or disgust. In all experimental conditions, HF HRV was correlated with activation of the right prefrontal cortex.

Thayer and Lane (2009) also elucidate hemispheric differences in cardiac activation. Aron et al. (2004) postulate that the right prefrontal cortex may have more potent input for cardiac modulation. This is not surprising, given the functional neurology of the right prefrontal cortex involved in emotional regulation and dysregulation. Nevertheless, the models propose that prefrontal activity will modulate cardiac output, including general heart rate and HRV. However, Mild TBI or concussion injuries are rarely lateralized, typically being more diffuse in etiology and MOI.

Williamson et al. (2013) propose a similar model. They propose a model of connections between the orbitofrontal cortex through the uncinate fasciculus to the amygdala, terminating in the sympathetic nervous system. The importance of these white matter tracts for this model is that they communicate from the prefrontal cortices to the brain stem, then to the heart. Disruption of the white matter tracts may induce a loss of inhibitory control upon theANS. Not only does this loss of inhibitory control result in impairment of cognitive abilities, but it also can result in loss of emotional regulation, as seen in soldiers and civilians with PTSD. This review cites similar neuroimaging studies to Thayer et al. (2009) as well as citing neurotransmitter involvement.

Studies using DTI assess the integrity of the white matter tracts, whereas typical MRI including T1, T2, and FLAIR protocols may not show neuropathology in white matter. In DTI studies, fractional anistrophy (FA) and mean diffusivity (MD) are measures of white matter integrity noted to be impaired in concussion. Disruption may result in loss of neural transmission or in reduction in transmission time due to neural deformation from physical trauma (Bazarian et al., 2007).

\section{MECHANISM OF INJURY IN CONCUSSION}

As noted above, children from pre-school to senior high school are most often injured in bicycle accidents. Among teen sports, American football is the next most frequent concussion generator. By gender in this age group, American football is the greatest concussion generator for males, while soccer is the greatest concussion generator for females. Both sports can provide blunt trauma at high velocity, either directly through head-to-head strikes, footor knee-to-head strikes, or striking the ground or another object, such as the goal. Due to the jagged cranial cavity, most often the physical injury to the brain impacts the orbitofrontal and anterior temporal areas. Linear forces are more often involved in headto-head contact of athletes and persons in motor vehicles with straight-line acceleration, then impact, followed by rapid deceleration. In contrast, rotational injuries may be seen in open-field tackles or checking or boarding on the ice of a hockey rink.

While traditional neuroimaging will not show white matter attenuation, a recent study of Division I Ivy League football players 
with subconcussive injuries (not diagnosed with a formal concussion) revealed changes in FA and MD DTI after a season of play (McAllister et al., 2014). The groups compared contact sports, such as American football and soccer versus non-contact sports, including cross country and track and field. There were significant changes in FA and MD in the contact sport group over the course of a season. Long-term follow up is needed to ascertain if these changes are reversible (some data points to this) or if they are non-reversible. However, this does validate the concerns about the integrity of white matter tracts as being the conduit between the heart and brain.

Thompson and Hagedorn (2012) report alterations in HRV in patients with concussion or mild TBI, including low amplitude and poor rhythmicity. Decrease in HRV has been seen among patients with all levels of TBI severity, from Severe TBI (Baugley et al., 2006) to concussion (Goldstein et al., 1998; La Fountaine etal., 2009). Disruption in cardiovascular reactivity was noted after sports concussion, with interruptions of middle cerebral artery blood velocity after oxidative stress (Len et al., 2011). Len et al. (2011) studied HRV in Canadian Junior Hockey League players with and without concussion and matched for demographic characteristics. In the resting condition, there were no differences noted. However, upon exertion, there were significant differences in total HRV, as well as LF and HF power for the concussed group. This has implications for elite athletes who quickly need to respond with greater cardiovascular output to perform well in their sport (Gall et al., 2004). By extension, this cardiovascular output dysfunction may also hold true for soldiers concussed in IED blasts. Similarly, Leddy et al. (2007) fully reviewed the ANS changes from concussion, including "greater sympathetic and lower parasympathetic activity ..." and cerebrovascular dysregulation. These concussive changes may affect multiple organ systems, including pulmonary, hepatic, and renal. They suggest an individualized aerobic exercise program that is below the threshold of symptom onset.

\section{HRV AND EEG}

As multiple models postulate a heart-brain connection in HRV, measurement can empirically validate this neurovisceral relationship. Searches of both Medline and PsychInfo revealed only a few studies that addressed measurement of EEG changes with HRV intervention. Sherlin et al. (2010) measured EEG variables during HRV training. They assessed 19-channel QEEG and sLORETA variables in a group of laboratory stressed-induced (non-injured) subjects undergoing HRV training. Significant changes in either alpha increase or beta decrease were found at Brodman's areas 24, 30 , and 31, all associated with the limbic system and cingulate gyrus. The authors postulate these measured EEG changes from HRV BFB reflect a decrease in autonomic arousal in brain areas critical for stress regulation.

Prinloo et al. (2013) examined EEG correlates of HRV intervention in stressed senior managers, further exposed to experimental laboratory stress. In this study, five EEG sites were monitored. Two frontal sites (Fp1 and Fp2) were monitored for muscular artifacts. The important midline sites of $\mathrm{Fz}, \mathrm{Cz}$, and $\mathrm{Pz}$ were monitored, as they are thought to reflect brain attention and arousal mechanisms. After a single session of HRV BFB, significant changes were found with reduced beta and increased theta at all three central sites. The authors suggest these EEG changes are reflective of "... increased relaxation, decreased anxiety and decreased mental effort ... (p. 31).” These EEG changes were associated with increases in LF and SDNN HRV variables.

Reid et al. (2013) assessed EEG changes in 40 clinical subjects, half of whom were athletes undergoing optimal performance training, including HRV BFB. For this study, only one EEG site was utilized: $\mathrm{Cz}$, the primary central site of the vertex, reflecting the sensorimotor rhythm (SMR) at 12-15 Hz. Clients showing successful HRV training (peak frequency heart rate between 0.05 and $0.15 \mathrm{~Hz}$ ) also showed significant increases in SMR amplitude. The authors conclude that the SMR increase reflects a state of relaxed anticipatory focus (Sterman, 1966) useful for athletes who need to perform better in stressful sport competitions, with greater flexibility and regulation over their ANS. By extension, soldiers might also benefit from this approach.

Collura (2009) developed a software and hardware protocol (BrainMaster, Bedford, OH, USA) that combined HRV and Alpha EEG training. Each parameter could be trained independently or simultaneously for greater HRV and Alpha enhancement. Bazanova et al. (2013) trained high Alpha EEG (10-12 Hz) to measure its effect on HRV. They found that healthy male subjects with low resting levels of Alpha who increased Alpha had lowered EMG, greater HRV and showed increases in cognitive performance. They also noted that these changes were not present in a control group that did not receive any feedback, thus validating the need for actual feedback for self-regulation.

To date, only case studies have been conducted on concussed patients using either HRV or Neurofeedback interventions. Lagos et al. (2013) used a 10-week HRV BFB protocol on an adult athlete with post-concussion syndrome. At the end of treatment, the patient showed greater HRV, and greater LF power, as well as significant reduction in severity of post-concussion headaches and overall post-concussive symptoms. Thompson et al. (2013) report the case of an athlete who received a sport-related concussion and was treated with a multi-modal intervention, including both HRV BFB and neurofeedback. Bhandari et al. (2013) used both HRV and neurofeedback to treat an adult male with a Severe TBI. While this patient was much more severely injured than a person with a concussion, after a lengthy course of treatment he showed improvement on multiple QEEG parameters, as well as three HRV parameters, and was able to function successfully in his personal and work life. Lagos et al. (2012) provide a comprehensive rationale for HRV BFB in prolonged PCS from hyperactivation of the sympathetic nervous system and hypoactivation of the parasympathetic nervous system. In addition to changes in HRV as an outcome measure for BFB intervention, they also recommend multimodal measures, such as cardiovascular and neurovegetative functioning, and quality of life indicators.

\section{NEUROCOGNITIVE ENHANCEMENT AND REHABILITATION WITH HRV BFB}

Several compelling studies have implicated HRV BFB in neurocognitive enhancement, particularly executive functioning and working memory. In studying non-injured persons, Hansen et al. (2003) divided military personnel on the basis of low or high HRV 
groups and then assessed sustained attention and working memory. As would be expected, the higher HRV group had superior performance on both measures. Other studies of executive functioning, including the ability to make decisions, plan, and benefit from feedback, have linked executive skills to high HRV. Murray and Russoniello (2012) divided university students between exercise or control groups. HRV including instantaneous changes of heart rate and spectral analysis of HRV were measured. Results indicated optimal performance with the peak inverted-U curve for both the complex Trail Making B task and a four-choice reaction timed complex test. Optimal neurocognitive performance was obtained in the LF band. These studies suggest that HRV training may be a viable intervention to promote executive function rehabilitation in PCS.

Collegiate basketball players with high-state anxiety levels were given ten minutes of HRV training for ten days to increase coherence and respiratory sinus arrhythmia (Paul and Garg, 2012). The premorbidly anxious collegiate basketball players showed improvement on objective measures of dribbling, passing, and shooting with increases in HRV, especially the LF range. This study has implications for HRV BFB training in the rehabilitation of autonomic and emotional dysregulation as a critical component in PCS recovery. In a study with military relevance (Saus et al., 2006), Norwegian Police Academy cadets with higher HRV were noted to have greater situational awareness, and to perform more accurately in complex shooting drills requiring focused attention and executive abilities. A compelling study not yet undertaken would be to investigate whether pre-deployment training in HRV could possibly reduce anxiety-related aspects of combat, including PTSD.

\section{SUMMARY AND CONCLUSION}

In summary, this article has attempted to elucidate the relationship between the brain, particularly the prefrontal cortices, the ANS, and the heart. There can be top-down or bottom-up interaction to both, such that the prefrontal cortices' judgment of ambiguity for threat attenuates HRV. Similarly, baseline higher levels of HRV, especially LF HRV, are associated with greater performance on complex neurocognitive tasks of concentration, working memory, and executive functioning, requiring prefrontal integrity. Further, as sports-related and perhaps military-related concussions interfere with optimal levels of HRV, interventions to restore or increase these optimal HRV levels are needed. Diet, endurance/cardiovascular exercise, and biofeedback are effective interventions. Of these three, HRV BFB can be accomplished with minimal hardware, software, time, cost, and effort constraints upon an individual over a period of a few days. Additionally, biofeedback provides real-time feedback for the attribute of interest, allowing the athlete or soldier to exercise cognitive control over their physiology.

Future research is needed to assess the EEG correlates of HRV intervention on randomized, controlled groups of symptomatic post-concussion athletes and soldiers. As Tan et al. (2013) noted, HRV intervention can be more acceptable for veterans with PTSD than the emotionally charged psychotherapies, and this same logic may hold true for athletes and soldiers with PCS. Data currently available regarding HRV BFB efficacy raise a compelling argument for the need for further empirical validation, not only for treatment of refractory sport and military concussions, but possibly predeployment stress inoculation training for soldiers, and pre-game training for athletes.

\section{ACKNOWLEDGMENTS}

The authors wish to sincerely thank Ms. Lauren Conder and the Library of the University of North Carolina at Chapel Hill for their research assistance.

\section{REFERENCES}

Abolfathi, N., Naik, A., Sotudeh Chafi, M., Karami, G., and Ziejewski, M. (2009). A micromechanical procedure for modelling the anisotropic mechanical properties of brain white matter. Comput. Methods Biomech. Biomed. Engin. 12, 249-262. doi: 10.1080/10255840802430587

Aron, A. R., Robbins, T. W., and Poldrack, R. A. (2004). Inhibition and the right inferior prefrontal cortex. Trends Cogn. Neurosci. 8, 170-177. doi: 10.1016/j.tics.2004.02.010

Barr, W. B., and McCrae, M. (2011). "Diagnosis and assessment of concussion," in The Handbook of Sport Neuropsychology, ed. F. M. Webbe (New York: Springer), 91-112.

Barr, W. B., Prichep, L. S., Chabot, R., Powell, M. R., and McCrea, M. (2012). Measuring brain electrical activity to track recovery from sport-related concussion. Brain Inj. 26, 58-66. doi: 10.3109/02699052.2011.608216

Baugley, I. J., Heriseanu, R. E., Felmingham, K. L., and Camerson, I. D. (2006). Dysautonomia and heart rate variability following severe traumatic brain injury. Brain Inj. 20, 437-444. doi: 10.1080/02699050600664715

Bazanova, O. M., Balioz, N. V., Mauravleva, K. B., and Skoraya, M. V. (2013). Effect of voluntary EEG Alpha power increase training on heart rate variability. Hum. Physiol. 39, 86-97. doi: 10.1134/S0362119712060035

Bazarian, J. J., Zhong, J., Blyth, B., Zhu, T., Kavicic, V., and Peterson, D. (2007). Diffusion tensor imaging detects clinically important axonal damage after mild traumatic brain injury: a pilot study. J. Neurotrauma 24, 1447-1459. doi: 10.1089/neu.2007.0241

Belanger, H. G., Kretzmer, T., Yoash-Gantz, R., Pickett, T., and Tupler, L. A. (2009). Cognitive sequelae of blast-related versus other mechanisms of brain injury. J. Int. Neuropsychol. Soc. 15, 1-8. doi: 10.1017/S1355617708090036

Bhandari, T., Thompson, L., and Reid-Chung, A. (2013). Treating postconcussion syndrome using neurofeedback: a case study. Biofeedback 41, 172-182. doi: 10.5298/1081-5937-41.4.03

Bigger, J. T., Fleiss, J. L., Steinman, R. C., Rolnitzky, L. M., Schneider, W. J., and Stein, P. K. (1995). R-R variability in healthy, middle-aged persons compared with patients with chronic coronary heart disease or recent acute myocardial infarction. Circulation 91, 1936-1943. doi: 10.1161/01.CIR.91.7.1936

Bigler, E. D. (2008). Neuropsychology and clinical neuroscience of persistent post-concussive syndrome. J. Int. Neuropsychol. Soc. 14, 1-22. doi: 10.1017/S135561770808017X

Blechert, J., Michael, T., Grossman, P., Lajtman, M., and Wilhelm, F. H. (2007). Autonomic and respiratory characteristics of post traumatic stress disorder and panic disorder. Psychosom. Med. 69, 935-943. doi: 10.1097/PSY.0b013e31815a8f6b

Bluml, S., and Brooks, W. M. (2006). "Magnetic resonanace spectroscopy of traumatic brain injury and concussion," in Foundations of Sport-Related Brain Injuries, eds S. Slobounov and W. Sebastianelli (New York, NY: Springer), 197-220. doi: 10.1007/0-387-32565-4_9

Broglio, S., Pontifex, M. B., O’Connor, P., and Hillman, C. H. (2009). The persistent effects of concussion on neuroelectric indices of attention. J. Neurotrauma 26, 1463-1470. doi: 10.1089/neu.2008.0766

Cernak, I., and Noble-Haeusslein, L. J. (2009). Traumatic brain injury: an overview of pathobiology with emphasis on military populations. J. Cereb. Blood Flow Metab. 30, 255-266. doi: 10.1038/jcbfm.2009.203

Collura, T. F. (2009). Combining EEG with heart rate training for brain/body optimization. Neuroconnections 1, 8-11.

Combatalade, D. (2010). Basics of Heart Rate Variability Applied to Psychophysiology. Montreal: Thought Technology.

Cooper, D. B., Chau, P. M., Armistead-Jehle, P., Vanderploeg, R. D., and Bowles, A. O. (2012). Relationship between mechanism of injury and neurocognitive 
functioning in OEF/OIF service members with mild traumatic brain injuries. Mil. Med. 177, 1157-1160. doi: 10.7205/MILMED-D-12-00098

Gall, B., Parkhouse, W., and Goodman, D. (2004). Heart rate variability of recently concussed athletes at rest and exercise. Med. Sci. Sports Exerc. 36, 1269-1274. doi: 10.1249/01.MSS.0000135787.73757.4D

Garner, J., and Brett, S. J. (2007). Mechanisms of injury by explosive devices. Anesthesiol. Clin. 25, 147-160. doi: 10.1016/j.anclin.2006.11.002

Gevirtz, R. (2013). The promise of heart rate variability biofeedback: evidence-based applications. Biofeedback 41, 110-120. doi: 10.5298/1081-5937-41.3.01

Gilchrist, J., Thomas, K. E., Xu, L., McGuire, L. C., and Coronado, V. G. (2011). Nonfatal sports and recreation related traumatic brain injuries among children and adolescents treated in emergency departments in the United States, 20012009. MMWR 60, 1337-1342.

Giza, C. G., and Hovda, D. A. (2001). The neurometabolic cascade of concussion. J. Athl. Train. 36, 228-235.

Goldstein, B., Toweill, D., Susanna, L., Sonnenthal, K., and Kimberly, B. (1998) Uncoupling of the autonomic and cardiovascular systems in acute brain injury. Am. J. Physiol. 275(4 Pt 2), R1287-R1292.

Guskiewicz, K. P., and Mihalik, J. P. (2011). Biomechanics of sport concussion: quest for the elusive injury threshold. Exerc. Sport Sci. Rev. 39, 4-11. doi: 10.1097/JES.0b013e318201f53e

Hansen, A. L., Johnsen, B. H., Sollers, J. J., Sand Thayer, J. F. (2004). Heart rate variability training and its relation to prefrontal cognitive function: the effects of training and detraining. Eur. J. Appl. Physiol. 93, 263-272. doi: 10.1007/s00421004-1208-0

Hansen, A. L., Johnsen, B. H., and Thayer, J. F. (2003). Vagal influence in the regulation of attention and working memory. Int. J. Psychophysiol. 48, 263-274. doi: 10.1016/S0167-8760(03)00073-4

Hautala, A. J., Kiviniemi, A. M., and Tulppo, M. (2009). Individual responses to aerobic exercise: the role of the autonomic nervous system. Neurosci. Biobehav. Rev. 33, 107-115. doi: 10.1016/j.neubiorev.2008.04.009

Hedelin, R., Bjerle, P., and Henriksson-Larsen, K. (2001). Heart rate variability in athletes: relationship with central and peripheral performance. Med. Sci. Sport Exerc. 33, 1394-1398. doi: 10.1097/00005768-200108000-00023

Kontos, A., Elbin, R., and Collins, M. (2006). "Aerobic fitness and concussions outcomes in high school football," in Foundations of Sport-Related Brain Injuries, eds S. Slobounov and W. Sebastianelli (New York, NY: Springer), 315-340. doi 10.1007/0-387-32565-4_14

La Fountaine, M. F., Heffernan, K. S., Gossett, J. D., Bauman, W. A., and De Meersman, R. E. (2009). Transient suppression of heart rate complexity in concussed athletes. Auton. Neurosci. 148, 101-103. doi: 10.1016/j.autneu.2009.03.001

Lagos, L., Bottiglieri, T., Vaschillo, B., and Vaschillo, E. (2012). Heart rate variability biofeedback for postconcussion syndrome: implications for treatment. Biofeedback 40, 150-153. doi: 10.5298/1081-5937-40.4.05

Lagos, L., Thompson, J., and Vaschillo, E. (2013). A preliminary study: heart rate variability biofeedback for treatment of postconcussive syndrome. Biofeedback 41, 136-143. doi: 10.5298/1081-5937-41.3.02

Lane, R. D., McRae, K., Reiman, E. M., Ahern, G. L., and Thayer, J. F. (2007). Neural correlates of vagal tone during emotion. Psychom. Med. 69, 1-8.

Leddy, J. J., Kozlowski, K., Fung, M., Pendergast, D. R., and Willer, B. (2007). Regulatory and autoregulatory physiological dysfunction as a primary characteristic of post concussion syndrome: implications for treatment. NeuroRehabilitation 22, 199-205.

Lehrer, P. (2013). History of heart rate variability biofeedback research: a personal and scientific voyage. Biofeedback 41, 88-97. doi: 10.5298/1081-5937-41. 3.03

Lehrer, P. M., Vaschillo, E., and Vaschillo, B. (2000). Resonant frequency biofeedback training to increase cardiac variability: rationale and manual for training. Appl. Psychophysiol. Biofeedback 25, 177-191. doi: 10.1023/A:1009554825745

Lehrer, P., Vaschillo, B., Zucker, T., Graves, J., Katsamanis, M., Aviles, M., et al. (2013). Protocol for heart rate variability biofeedback training. Biofeedback 41, 98-109. doi: 10.5298/1081-5937-41.3.08

Len, T., Nearly, J., Asmundson, G., Goodman, D., Bjornson, B., and Bhambhani, Y. (2011). Cerebrovascular reactivity impairment after sport-induced concussion. Med. Sci. Sports Exerc. 43 , 2241-2248. doi: 10.1249/MSS.0b013e3182249539

Levin, H. S., Li, X., McCauley, S. R., Hanten, G.,Wilde, E. A., and Swan, P. (2012). Neuropsychological outcome of $\mathrm{mTBI}$ : a principal components analysis approach. J. Neurotrauma 30, 625-632. doi: 10.1089/neu.2012.2627
Lima-Silva, A. E., Bertuzzi, R., Dalquano, E., Nogueira, M., Casarini, D., Kiss, M. A., et al. (2010). Influence of high- and low-carbohydrate diet following glycogendepleting exercise on heart rate variability and plasma catecholamines. Appl. Physiol. Nutr. Metab. 35, 541-547. doi: 10.1139/H10-043

Lippa, S. M., Pastorek, N. J., Benge, J. F., and Thornton, G. M. (2010). Postconcussive symptoms after blast and nonblast-related mild traumatic brain injuries in Afghanistan and Iraq war veterans. J. Int. Neuropsychol. Soc. 16, 856-866. doi: 10.1017/S1355617710000743

McAllister, T. W., Ford, J. C., Flashman, L. A., Maerlender, A., Greenwald, R. M., Beckwith, J. G., et al. (2014). Effects of head impacts on diffusivity measures in a cohort of collegiate contact sport athletes. Neurology 82, 1-7. doi: 10.1212/01.wnl.0000438220.16190.42

McCrea, M., Prichep, L., Powell, M. R., Chabot, R., and Barr, W. B. (2010). Acute effects and recovery after sport-related concussion: a neurocognitive and quantitative brain electrical activity study. J. Head Trauma Rehabil. 25, 283-292. doi: 10.1097/HTR.0b013e3181e67923

McCrory, P., Meeuwisse, W., Aubry, M., Cantu, B., Dvorák, J., Echemendia, R. J., et al. (2013). Consensus statement on concussion in sport: the 4th International conference on concussion in sport held in Zurich, November 2012. Br. J. Sports Med. 47, 250-258. doi: 10.1136/bjsports-2013-092313

Moss, D., Lagos, L., and Shaffer, F. (2013). Don't add or miss a beat: a special issue on current evidence and current practice in heart rate variability biofeedback. Biofeedback 41, 83-84. doi: 10.5298/1081-5937-41.3.09

Murray, N. P., and Russoniello, C. (2012). Acute physical activity on cognitive function: a heart rate variability examination. Appl. Psychophysiol. Biofeedback 37, 219-227. doi: 10.1007/s10484-012-9196-z

Palma, J., and Benarroch, E. E. (2014). Neural control of the heart. Neurology 83, 261-271. doi: 10.1212/WNL.0000000000000605

Pardini, J., Henry, L. C., and Fazio, V. C. (2011). "Neuroimaging techniques and sports-related concussion," in The Handbook of Sport Neuropsychology, ed. F. M. Webbe (New York: Springer), 113-130.

Paul, M., and Garg, K. (2012). The effect of heart rate variability biofeedback on performance psychology of basketball players. Appl. Psychophysiol. Biofeedback 37, 131-144. doi: 10.1007/s10484-012-9185-2

Ponsford, J., Willmott, C., Rothwell, A., Cameron, P., Kelly, A., Nelms, R., et al. (2000). Factors influencing outcome following mild traumatic brain injury in adults. J. Int. Neuropsychol. Soc. 6, 568-579. doi: 10.1017/S1355617700655066

Prinloo, G. E., Rauch, H. G., Karpul, D., and Derman, W. E. (2013). The effect of a single session of short duration heart rate variability biofeedback on EEG: a pilot study. Appl. Psychophysiol. Biofeedback 38, 45-56. doi: 10.1007/s10484-0129207-0

Reid, A., Nihon, S., Thompson, L., and Thompson, M. (2013). The effects of heart rate variability training on sensorimotor rhythm: a pilot study. J. Neurother. 17, 43-48. doi: 10.1080/10874208.2013.759020

Saus, E. R., Johnsen, B. H., Eid, J., Riisem, P. K., Andersen, R., and Thayer, J. (2006). The effect of brief situational awareness training in a police shooting simulator: an experimental study. Mil. Psychol. 18,3-21. doi: 10.1207/s15327876mp1803s_2

Sherlin, L., Muench, F., and Wyckoff, S. (2010). Respiratory sinus arrhythmia feedback in a stressed population exposed to a brief stressor demonstrated by quantitative EEG and sLORETA. Appl. Psychophysiol. Biofeedback 35, 219-228. doi: 10.1007/s10484-010-9132-z

Sterman, B. (1966). Physiological origins and functional correlates of EEG rhythmic activities: implications for self-regulation. Biofeedback Self Regul. 21, 3-33. doi: 10.1007/BF02214147

Tan, G., Fink, B., Dao, T. K., Hebert, R., Farmer, L. S., Sanders, A., et al. (2009). Associations among Pain, PTSD, mTBI, and heart rate variability in veterans of operation enduring and Iraqi freedom: a pilot study. Pain Med. 10, 1237-1245. doi: 10.1111/j.1526-4637.2009.00712.x

Tan, G., Wang, P., and Ginsberg, J. (2013). Heart rate variability and post traumatic stress disorder. Biofeedback 41, 131-135. doi: 10.5298/1081-5937-41.3.05

Teasdale, G., and Jeanette, B. (1974). Assessment of coma and impaired consciousness: a practical scale. Lancet 2, 81-84. doi: 10.1016/S0140-6736(74)91639-0

Thayer, J. F., Ahs, F., Fredirkson, M., Sollers, J. J., and Wager, T. D. (2012). A metaanalysis of heart rate variability and neuroimaging studies: implications for heart rate variability as a marker for stress and health. Neurosci. Biobehav. Rev. 36, 747-756. doi: 10.1016/j.neubiorev.2011.11.009

Thayer, J. F., Hansen, A. L., Saus-Rose, E., and Johnsen, B. H. (2009). Heart rate variability, prefrontal neural function, and cognitive performance: the neurovisceral 
integration perspective on self-regulation, adaptation, and health. Ann. Behav. Med. 37, 141-153. doi: 10.1007/s12160-009-9101-z

Thayer, J. F., and Lane, R. D. (2009). Claude Bernard and the heart-brain connection: further elaboration of a model of neurovisceral integration. Neurosci. Biobehav. Rev. 33, 81-88. doi: 10.1016/j.neubiorev.2008.08.004

Thompson, J., and Hagedorn, D. (2012). Multimodal analysis: new approaches to the concussion conundrum. J. Clin. Sport Psychol. 6, 22-46.

Thompson, M., Thompson, L., Reid-Chung, A., and Thompson, J. (2013). Managing traumatic brain injury: appropriate assessment and a rationale for using neurofeedback and biofeedback to enhance recovery in postconcussion syndrome. Biofeedback 41, 158-173. doi: 10.5298/1081-5937-41.4.07

Williamson, J. B., Heilman, K. M., Porges, E. C., Lamb, D. G., and Porges, S. W. (2013). A possible mechanism for PTSD symptoms in patients with traumatic brain injury: central autonomic network disruption. Front. Neuroeng. 6:13. doi: 10.3389/fneng.2013.00013
Conflict of Interest Statement: The authors declare that the research was conducted in the absence of any commercial or financial relationships that could be construed as a potential conflict of interest.

Received: 01 June 2014; accepted: 27 July 2014; published online: 13 August 2014. Citation: Conder RL and Conder AA (2014) Heart rate variability interventions for concussion and rehabilitation. Front. Psychol. 5:890. doi: 10.3389/fpsyg.2014.00890

This article was submitted to Psychology for Clinical Settings, a section of the journal Frontiers in Psychology.

Copyright (C) 2014 Conder and Conder. This is an open-access article distributed under the terms of the Creative Commons Attribution License (CC BY). The use, distribution or reproduction in other forums is permitted, provided the original author(s) or licensor are credited and that the original publication in this journal is cited, in accordance with accepted academic practice. No use, distribution or reproduction is permitted which does not comply with these terms. 